\title{
The Diagnostic Performance of 68Ga-PET or PET/CT in Neuroendocrine Tumour, A Meta Analysis
}

Xue Liu ${ }^{1 *}$, Qilian Ran ${ }^{2}$, Zhixiao Wei ${ }^{3}$, Shiliang Zhou ${ }^{1}$, Tao Jiang ${ }^{1}$, Jiang $\mathbf{W u}^{1}$ and Zhen Shu ${ }^{1}$

${ }^{1}$ Department of Nuclear Medicine, The First People's Hospital of Huaihua, Hunan, 418000, China

${ }^{2}$ Department of General Surgery, The First Affiliated Hospital of Hunan Medical College, Huaihua, Hunan, 418000, China

${ }^{3}$ Department of Nuclear Medicine, The First Affiliated Hospital of Guangxi Medical University, Nanning, Gaungxi 530000, China

\begin{abstract}
Objective: To evaluate the diagnostic performance of $68 \mathrm{Ga}-\mathrm{PET}$ or PET/CT in neuroendocrine tumour (NET) with a meta-analysis.

Methods: The major electronic medical databases, PudMed,The cochrance Library, Embase.com, Web of Science, were searched for relevant papers up to February 2018. According to the inclusion and exclusion criteria, the eligible literature was graded with the Quality Assessment of Diagnostic Accuracy Studies (QUADAS). Pooled sensitivity specificity Diagnostic odds ratio DOR and Summary Receiver Operating Characteristics (SROC) and the areas under the curve AUC were calculated with Meta-Disc 1.4 software. The publication bias was assessed with Stata 12.0 software.
\end{abstract}

Results: A total of 14 studies were finally included. The pooled sensitivity, specificity, positive likelihood ratio, negative likelihood ratio and diagnostic odds ratio of $68 \mathrm{Ga}-\mathrm{PET}$ or PET/CT were $90.4 \%(95 \% \mathrm{Cl}, 87.5 \%$ 92.8\%),84.2\% 95\% Cl 79.3\%-088.3\%, 4.646 95\% Cl,3.222-6.699, 0.135 95\% Cl,0.095-0.192 and 41.082 95\% $\mathrm{Cl}, 23.114-73.018$, respectively. The AUC and $\mathrm{Q}^{*}$ indexes of the SROC were 0.9319 and 0.8674 , respectively. Deek's funnel plot shows symmetry $(P=0.48)$, and Begg's and Egger's test results indicating that there was no publication bias.

Conclusion: This meta-analysis suggested that $68 \mathrm{Ga}-\mathrm{PET}$ or PET/CT have higher sensitivity and specificity by detecting primary and metastatic lesions to diagnose neuroendocrine tumour.

Keywords: 68Ga; PET; Diagnosis; Neuroendocrine tumour; Metaanalysis

\section{Introduction}

Neuroendocrine tumors (NETs) is a kind of mainly distributed in the lung, gastrointestinal tract and pancreas organs, and it is rare and slow-growing tumours. Some studies have shown that the incidence of NETs is increasing year by year, it depends on the diagnostic technology advances and the gradual improvement of the people understanding of disease [1]. The correct staging and grading for the NETs by clinicians can lead to a five-year survival rate of about $75 \%$. After early detection, surgical treatment can be performed. In fact, it often loses the best chance of surgical resection for NETs [1]. Therefore, it is very important to accurately diagnose and provide appropriate treatment for the disease in the early stage. Due to various factors, such as tumour size, location of lesions and metabolism, traditional imaging methods, such as CT, ultrasound and magnetic resonance imaging, have low detection rate for NETs. With the development of medical technology, the $68 \mathrm{Ga}$ labelling of somatostatin receptor in nuclear medical imaging technology has led to the gradual application of PET in the diagnosis, treatment and management of neuroendocrine tumours [2]. At present, 68Ga-DOTATATE, 68Ga-DOTA-TOC and 68Ga-DOTA-NOC are the most widely used clinical researches [2]. However, due to differences in geographical indications, indications and sample size, the conclusion of published research has inconsistent in evaluating the value of $68 \mathrm{Ga}$-PET imaging. Therefore, this meta-analysis is used to analyse the diagnostic value of $68 \mathrm{Ga}-\mathrm{PET}$ or PET/CT imaging in neuroendocrine tumours.

\section{Materials and Methods}

\section{Inclusion and Exclusion Criteria}

Inclusion criteria: (1) The literature used 68ga-pet or PET/CT imaging diagnosis NETs. (2) The subjects were diagnosed as NETs by pathology or clinical follow-up. (3) The literature data can calculate four-fold table data, this is true positive. (TP), false positive ( FP), false negative (FN)) and true negative (TN). (4) The literature was analyzed by studying the number of patients not lesions.

Exclusion criteria: (1) Review, conference papers, case reports and repeated studies. (2) Unable to access full text.

\section{Literature search}

The English literature published were researched by two evaluators in database of PudMed, The cochrance Library, Embase.com, and Web of Science form the establishment of the database to February 2018. At the same time, it also tracks the references of newly published system evaluation or meta analysis.Researching words: (1) "68Ga OR Ga68 OR Ga-68 OR Ga OR gallium” and (2) “"PET” OR "positron emission tomography" OR "positron emission tomography/computed tomography" OR "PET/CT" OR "positron emission tomographycomputed tomography" OR "PET-CT"” and (3) “"Neuroendocrine tumors" OR"NETs"”.

*Corresponding author: Xue Liu, Department of Nuclear Medicine, The First People's Hospital of Huaihua, Hunan 418000, China, Tel: 18377102453; E-mail: yxliuxue@foxmail.com

Received May 16, 2018; Accepted May 25, 2018; Published June 04, 2018

Citation: Liu X, Ran Q, Wei Z, Zhou S, Jiang T, et al. (2018) The Diagnostic Performance of 68Ga-PET or PET/CT in Neuroendocrine Tumour, A Meta Analysis. J Nucl Med Radiat Ther 9: 364. doi: 10.4172/2155-9619.1000364

Copyright: (c) 2018 Liu X, et al. This is an open-access article distributed unde the terms of the Creative Commons Attribution License, which permits unrestricted use, distribution, and reproduction in any medium, provided the original author and source are credited. 


\section{Literature selection and data extraction}

The retrieved literature was imported into the document manager Endnote X7 to remove duplicate references. Two evaluators independently select the literatures according to the prior setting inclusion and exclusion criteria, and finally determine the qualified studies. If the opinion is not consistent, through negotiation or third party discussion. The basic characteristics of included study are as follows: Gender, age, author, published year, country, type of study (prospective or retrospective), diagnostic criteria for disease, sample size, blind method, type of imaging agent (68Ga-DOTA-TATE, 68GaDOTA-TOC or 68Ga-DOTA-NOC).

\section{Literature quality evaluation}

The Quality Assessment of Diagnostic Accuracy Studies (QUADAS) [3], recommended by the Cochrane collaborative network was used to assess the Quality of the included studies. Deletion nonobligatory items of 3, 8 and 9 items and determine ultimately the evaluation criteria 11 items. Each item is evaluated with "yes", "no" or "not clear".

\section{Statistical analysis}

Meta-analysis was performed using meta-disc 1.4 software [4]. The threshold effect was tested by ROC curve and Spearman correlation coefficient. If threshold effect exists, the best way to pool data is to fit the Summary Receiver Operating Characteristics (SROC) curve and calculate areas under the curve (AUC).If no threshold effect, we will calculate the combined sensitivity (Sen), specifity (Spe), Positive likelihood thewire (LR+) and Negative likelihood thewire ( LR-) and Diagnostic odds ratio (DOR). Meanwhile, meta-regression analysis was used to find the heterogeneity source. Deek's funnel plot analysis was conducted with Stata 12.0 software to detect publish bias (Table 1).

\section{Results}

\section{Included literature}

A total of 752 literatures were retrieved, the title and abstract were reviewed, and 108 were left out of the literatures that were significantly unrelated to the subject and repeated. After careful reading, the reasons for the exclusion of 90 articles were: (1) 32 of 90 were excluded because of review, case report, editor's letter and animal experiment 32; (2) 18 articles were unable to extract the complete four table data, the literatures only reported the sensitivity and no specificity data; (3) 40 artcles only evaluated $68 \mathrm{Ga}$-PET or PET/CT for the management of disease staging and not evaluated diagnosis performance. Finally, 14 papers were included.

\section{The basic features of the included literature}

There were seven studies of prospective [5-11] and retrospective [12-18], respectively. Three articles [5,7,11] imaging instruments were PET, and 11 were PET/CT [6-8], [10-12], [12-18]. Eight [6-9,10,1317] articles study methods were blinded and another six articles $[5,7,8,11,12,18]$ not given. Eight studies [5,7-10,15-17] photographic developer were $68 \mathrm{Ga}$-DOTATOC and four studies $[11,12,14,18]$ were 68Ga-DOTATATE and two studies $[6,13]$ were $68 \mathrm{Ga}$-DOTANOC.

\section{The quality evaluation of the included literature}

This study used the QUADAS tool to evaluate the quality. Six articles had nine questions answered "yes", another two questions answered "no or unclear". Eight articles had eight questions answered "yes", another three questions answered "no or unclear".

\begin{tabular}{|c|c|c|c|c|c|c|c|c|c|c|}
\hline \multirow[b]{2}{*}{ Author } & \multirow[b]{2}{*}{ Year } & \multirow[b]{2}{*}{ Device } & \multirow[b]{2}{*}{ design type } & \multirow[b]{2}{*}{ age } & \multirow[b]{2}{*}{ imaging agent } & \multirow[b]{2}{*}{$68 \mathrm{Ga}$ dose } & \multirow[b]{2}{*}{ blind } & \multirow[b]{2}{*}{ golden standard } & \multicolumn{2}{|c|}{ QUADAS } \\
\hline & & & & & & & & & yes & $\begin{array}{l}\text { No or } \\
\text { unclear }\end{array}$ \\
\hline Gabriel et al & 2007 & PET & Prospective & 58.2 & $\begin{array}{l}\text { 68Ga- } \\
\text { DOTATOC }\end{array}$ & $\begin{array}{c}100-150 \\
\mathrm{MBq}\end{array}$ & NG & histopathology,imaging, follow-up & 9 & 2 \\
\hline Buchmann et al & 2007 & PET & Prospective & 52 & $\begin{array}{l}\text { 68Ga- } \\
\text { DOTATOC }\end{array}$ & $\begin{array}{l}100-228 \\
\mathrm{MBq}\end{array}$ & NG & histopathology & 8 & 3 \\
\hline Versari et al & 2010 & PET-CT & Retrospective & 56 & $\begin{array}{l}\text { 68Ga- } \\
\text { DOTATOC }\end{array}$ & $\begin{array}{c}1.5-2 \mathrm{MBq} / \\
\mathrm{Kg}\end{array}$ & yes & fine needle biopsy and/or surgery & 9 & 2 \\
\hline Ruf et al & 2011 & PET-CT & Retrospective & 57 & $\begin{array}{l}\text { 68Ga- } \\
\text { DOTATOC }\end{array}$ & $\begin{array}{c}100-120 \\
\mathrm{MBq}\end{array}$ & yes & $\begin{array}{l}\text { clinical and imaging follow-up, } \\
\text { histopathology }\end{array}$ & 9 & 2 \\
\hline Naswa et al & 2011 & $\mathrm{PET} / \mathrm{CT}$ & Prospective & 50 & $\begin{array}{c}\text { 68Ga- } \\
\text { DOTANOC }\end{array}$ & $\begin{array}{c}132-222 \\
\mathrm{MBq}\end{array}$ & yes & histopathology & 9 & 2 \\
\hline Lapińska et al & 2011 & $\mathrm{PET} / \mathrm{CT}$ & Retrospective & 54 & $\begin{array}{c}\text { 68Ga- } \\
\text { DOTATATE }\end{array}$ & $\begin{array}{c}111-185 \\
\mathrm{MBq}\end{array}$ & NG & histopathology & 8 & 3 \\
\hline $\begin{array}{l}\text { Mayerhoefer } \\
\text { et al }\end{array}$ & 2012 & PET-CT & Retrospective & 61.9 & $\begin{array}{c}\text { 69Ga- } \\
\text { DOTATOC }\end{array}$ & $150 \mathrm{MBq}$ & yes & histopathology, imaging examinations & 8 & 3 \\
\hline Haug et al & 2012 & $\mathrm{PET} / \mathrm{CT}$ & Retrospective & 58 & $\begin{array}{c}\text { 68Ga- } \\
\text { DOTATATE }\end{array}$ & $200 \mathrm{MBq}$ & NG & histopathology or clinical follow-up & 8 & 3 \\
\hline $\begin{array}{l}\text { Beiderwellen } \\
\text { et al }\end{array}$ & 2013 & PET-CT & Prospective & 54 & $\begin{array}{c}\text { 68Ga- } \\
\text { DOTATOC }\end{array}$ & $51 \pm 11 \mathrm{MBq}$ & NG & histopathology & 9 & 2 \\
\hline Schraml et al & 2013 & PET-CT & Prospective & 57 & $\begin{array}{l}\text { 68Ga- } \\
\text { DOTATOC }\end{array}$ & $150 \mathrm{MBq}$ & yes & histopathology & 8 & 3 \\
\hline $\begin{array}{l}\text { Venkitaraman } \\
\text { et al }\end{array}$ & 2014 & PET-CT & Prospective & 34.22 & $\begin{array}{l}\text { 68Ga- } \\
\text { DOTATOC }\end{array}$ & 74-111 MBq & yes & histopathology & 9 & 2 \\
\hline $\begin{array}{l}\text { Srirajaskanth } \\
\text { et al }\end{array}$ & 2010 & PET & prospective & 55.5 & $\begin{array}{c}\text { 68Ga- } \\
\text { DOTATATE }\end{array}$ & $\begin{array}{c}120-200 \\
\mathrm{MBq}\end{array}$ & NG & histopathology & 8 & 3 \\
\hline Naswa etal & 2014 & $\mathrm{PET} / \mathrm{CT}$ & Retrospective & 48.8 & $\begin{array}{c}\text { 68Ga- } \\
\text { DOTANOC }\end{array}$ & $\begin{array}{c}132-222 \\
\mathrm{MBq}\end{array}$ & yes & histopathology & 8 & 3 \\
\hline Haug et al & 2014 & $\mathrm{PET} / \mathrm{CT}$ & Retrospective & 58 & $\begin{array}{c}\text { 68Ga- } \\
\text { DOTATATE }\end{array}$ & $200 \mathrm{MBq}$ & yes & histopathology & 8 & 3 \\
\hline
\end{tabular}

NG:not given,QUADAS:Quality Assessment of Diagnostic Accuracy Studies. 


\section{The results of meta-analysis}

According to the result of SROC curve plane scatter diagram and Spearman correlation coefficient is 0.152 , there were no threshold effect. $(\mathrm{P}=0.604)$. Further to analyze the other sources of method heterogeneity. We have calculated the heterogeneity of Sen, Spe, LR+, LR and DOR and the heterogeneity of sensitivity indicated that there was a slight heterogeneity between the studies ( $2=56.1 \%, \mathrm{P}=0.0054)$. Therefore, meta regression was conducted to explore the heterogeneity sources, and the main analysis of variables related to the study (such as research type, research design, blind method, imaging instruments and photographic developer, etc.). The results showed that the heterogeneity of this study was related to the blind method of the study design, namely, the accuracy of the blind method was 3.89 higher than that of the non-blind method [RDOR=3.89 (95\%CI,1.05-14.44), $\mathrm{P}=0.047$ ].

\section{Summery statistic}

Because of mild heterogeneity, the random effects model was used to quantitative combined weighted the result of Sen $90.4 \%$ (95\% CI, 87.5\%-92.8\%), Spe 84.2\% (95\% CI, 79.3\%-088.3\%), LR+ 4.646 (95\% CI, 3.222-6.699), LR- 0.135 (95\% CI, 0.095-0.192) and DOR 41.082 (95\% CI, 23-114 73.018), respectively (Figures 1-3). According to the SROC, the AUC and $\mathrm{Q}^{*}$ indexes of curves are 0.9319 and 0.8674 , respectively (Figure 4).

\section{Publication bias}

The Deek 'funnel plot drawn by Stata 12.0 software shows the basic symmetry of the graph, indicating that there is no publication bias $(\mathrm{P}=0.48)$ (Figure 5).

\section{Discussions}

The uniqueness of neuroendocrine tumor is the overexpression of somatostatin receptor in tumor cells, which provides a reliable basis for imaging diagnosis and peptide receptor nuclide therapy [2]. Octreotide marked In-111 is the first recognized somatostatin receptor to be used to diagnose neuroendocrine tumors, which was approved for release In the United States in June 1994 [19]. Although the Octreotide of imaging agent successfully used to diagnose NETs more than 20 years, but it needed relatively high gamma ray energy to complete imaging (171 kev-245 kev), and need use the medium energy probe to scan, and its image spatial resolution is low. At the end of 1990 there were 68Ga- DOTA imaging agent were applied to clinical, the tumor cells in one hour after the rapid uptake can quickly complete PET imaging after injection in patients, and improve the image quality and spatial resolution [2]. This study collected publicly published literature on the $68 \mathrm{Ga}$ PET or PET/CT and evaluated its diagnostic accuracy in NETs using a meta-analysis.

In this study, the results of meta-analysis were carried out and indicated that the $68 \mathrm{Ga}$ PET or PET/CT had higher accuracy in diagnosis of NETs, with a pooled sensitivity and specificity of $90.4 \%$ and $84.2 \%$, respectively. In addition, the DOR is 41.082 and the AUC and $\mathrm{Q}^{*}$ indices of SROC is 0.9319 and 0.8674 , and further indicated that the $68 \mathrm{Ga}$ PET or PET/CT had higher accuracy in NETs.

Some researchers [20], analyzed the application value of $68 \mathrm{Ga}$ DOTATOC PET imaging in NETs with meta-analysis. They analyzed the photographic developer of 68Ga-DOTATOC in diagnosing and changing clinical management decisions for NETs, and the sensitivity and specificity were 92\% (95\% CI, 85\%-96\%), 82\% (95\% CI,69\%-90\%), respectively. Howerver, the heterogeneity of the inclusion literature were not analyzed in their study. Treglia et al. [21] analyzed also the diagnostic efficacy of $68 \mathrm{Ga}$ PET or PET/CT in NETs. Their study had included 16 literatures, but only 5 literatures could extract the complete four table data. The sensitivity and specificity of the combination were 93\% (95\% CI: 91-95\%) and 91\% (95\% CI: 82-97\%), respectively. The heterogeneity was too high in their study, although the meta-regression analysis failed to find the source of heterogeneity.

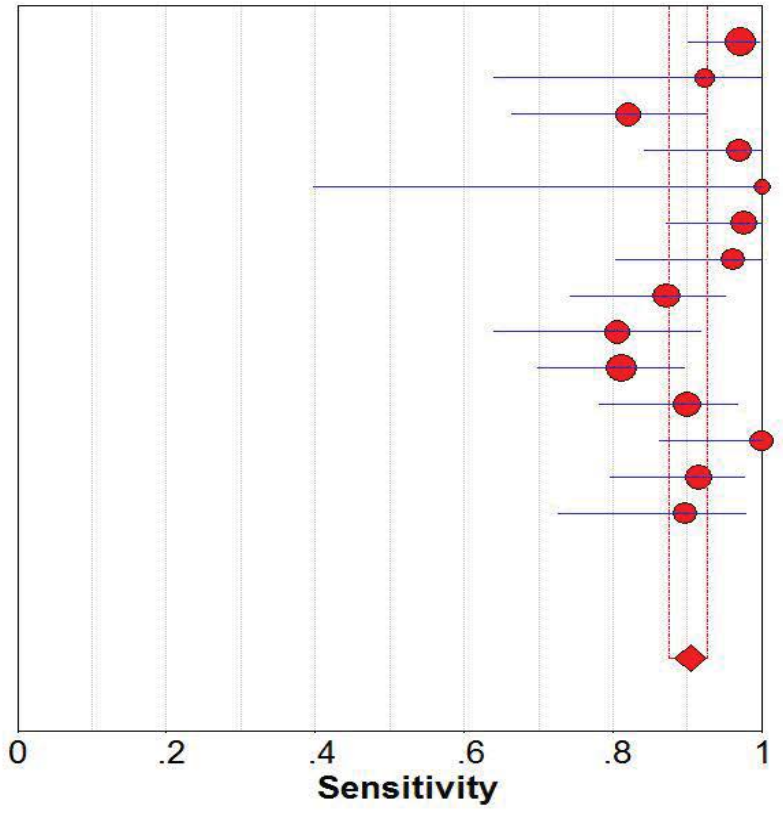

Sensitivity $(95 \% \mathrm{CI})$

Gabriel et al

Versari et al

Ruf et al

Mayerhoefer et al

Beiderwellen et al

Schraml et al

Venkitaraman et al

Srirajaskanthan et al

Haug et al

Naswa et al

?apińska et al

Buchmann et al

Naswa etal

Haug et al

Pooled Sensitivity $=0.90$ (0.88 to 0.93 )

Chi-square $=29.59 ; \mathrm{df}=13(\mathrm{p}=0.0054)$

Inconsistency $(\mathrm{I}$-square $)=56.1 \%$
0.97

0.92

0.82

0.97

1.00

0.98

0.96

0.87

0.81

0.81

0.90

1.00

0.91

0.90
$(0.64-1.00)$

$(0.66-0.92)$

$(0.84-1.00)$

$(0.40-1.00)$

$(0.87-1.00)$

$(0.80-1.00)$

$(0.74-0.95)$

$(0.64-0.92)$

$(0.70-0.90)$

$(0.78-0.97)$

$(0.86-1.00)$

$(0.80-0.98)$

$(0.73-0.98)$

Figure 1: The pooled sensitivity of $68 \mathrm{Ga}$ PET or PET-CT in NETs. 


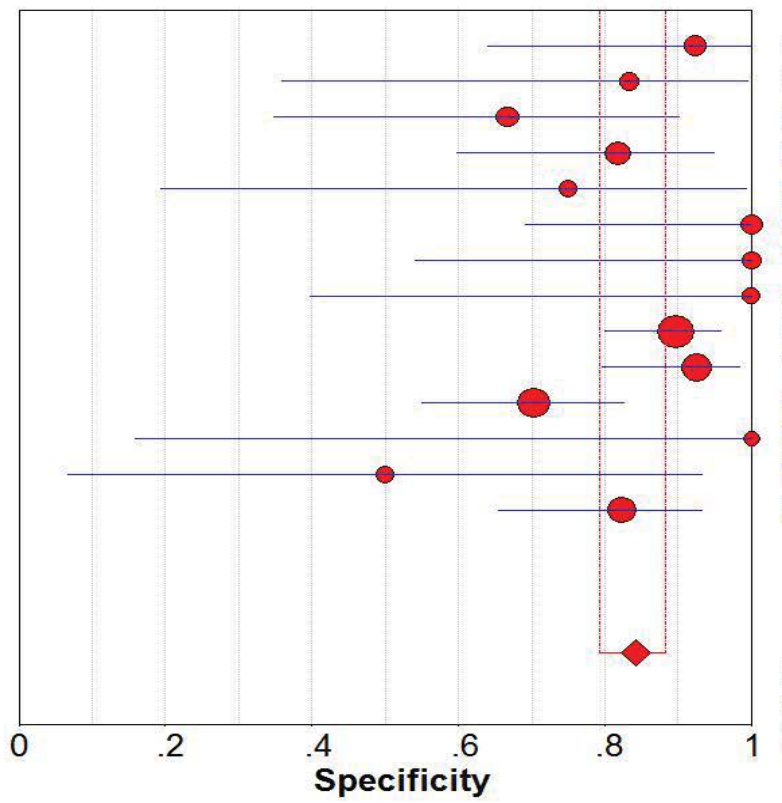

Specificity $(95 \% \mathrm{Cl})$

Gabriel et al

Versari et al

Ruf et al

Mayerhoefer et al

Beiderwellen et al

Schraml et al

Venkitaraman et al

Srirajaskanthan et al

Haug et al

Naswa et al

?apińska et al

Buchmann et al

Naswa etal

Haug et al

Pooled Specificity $=0.84(0.79$ to 0.88$)$

Chi-square $=23.48 ; d f=13(p=0.0363)$

Inconsistency (I-square) $=44.6 \%$

Figure 2: The pooled specitivity of $68 \mathrm{Ga}$ PET or PET-CT in NETs.

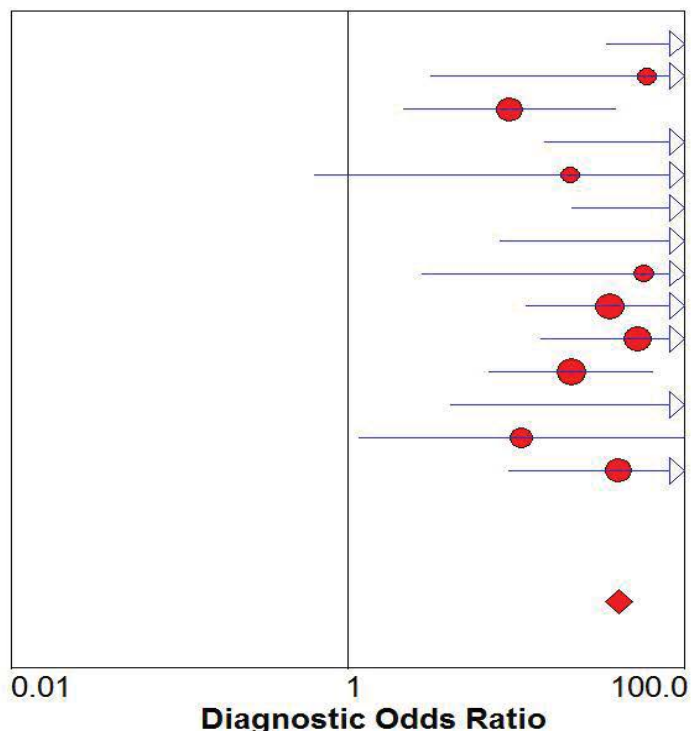

Gabriel et al Versari et al

Ruf et al

Mayerhoefer et al

Beiderwellen et al

Schraml et al

Venkitaraman et al

Srirajaskanthan et al

Haug et al

Naswa et al

?apińska et al

Buchmann et al

Naswa etal

Haug et al

\section{Diagnostic OR (95\% Cl)}

$414.00 \quad(34.76-4,931.35)$

$60.00 \quad(3.10-1,159.79)$

$9.14 \quad(2.14-39.07)$

$144.00(14.93-1,388.44)$

$21.00 \quad(0.64-689.99)$

$567.00 \quad(21.51-14,945.37)$

$221.00 \quad(8.03-6,079.12)$

$57.46 \quad(2.76-1,197.02)$

$36.10(11.58-112.56)$

$53.13(14.16-199.33)$

$21.21 \quad(6.95-64.73)$

$255.00 \quad(4.10-15,848.21)$

$10.75(1.18-98.15)$

$40.44 \quad(9.16-178.59)$

\section{Random Effects Mode}

Pooled Diagnostic Odds Ratio $=41.08$ (23.11 to 73.02)

Cochran- $Q=15.97 ; \mathrm{df}=13(\mathrm{p}=0.2506)$

Inconsistency (I-square) $=18.6 \%$

Tau-squared $=0.2109$

Figure 3: The pooled diagnostic odds ratio of $68 \mathrm{Ga}$ PET or PET-CT in NETs.

In our study, the pooled sensitivity and specificity were $90.4 \%$ and $84.2 \%$, roughly the same as other results. It is well to be reminded that our study have included three imaging agents of $68 \mathrm{Ga}$-DOTATOC, 68Ga-DOTATATE and 68Ga-DOTANOC and each article can extract the complete four table data, and this study discussed the heterogeneity test and heterogeneity source.
To discuss the source heterogeneity is an important part of meta analysis. The resuts of our study indicated that there are slight heterogeneity in this meta anlysis. It is important that we found the blind is the source of heterogeneity, and the accuracy of the blind method was 3.89 times higher than that non-blind method. Publication 
Citation: Liu X, Ran Q, Wei Z, Zhou S, Jiang T, et al. (2018) The Diagnostic Performance of 68Ga-PET or PET/CT in Neuroendocrine Tumour, A Meta Analysis. J Nucl Med Radiat Ther 9: 364. doi: 10.4172/2155-9619.1000364

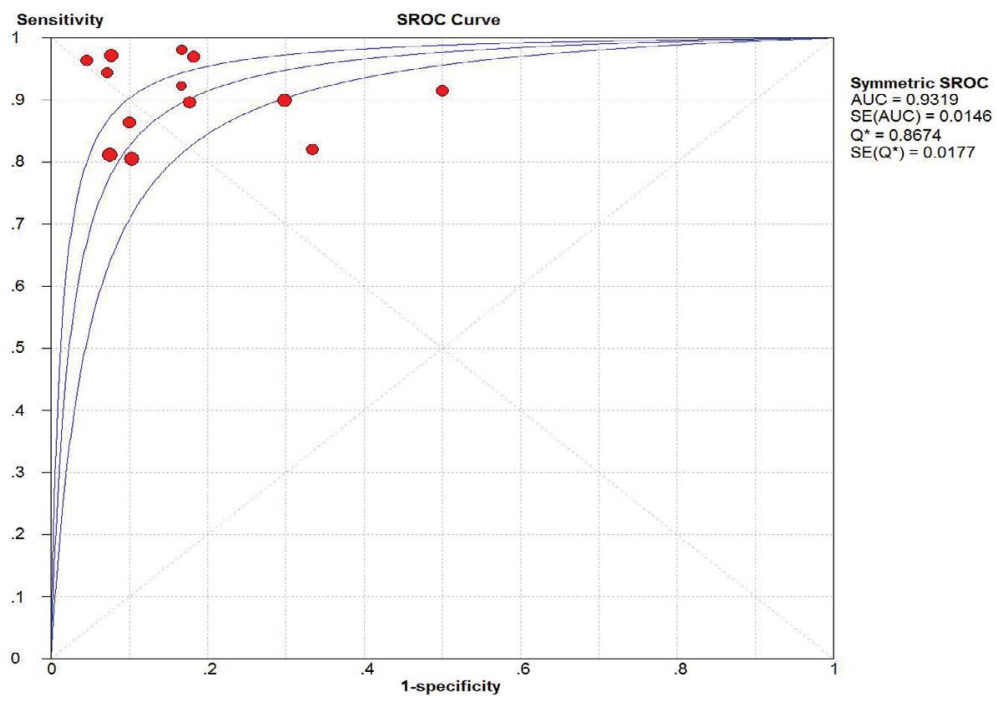

Figure 4: The SROC curve of $68 \mathrm{Ga}$ PET or PET-CT in NETs.

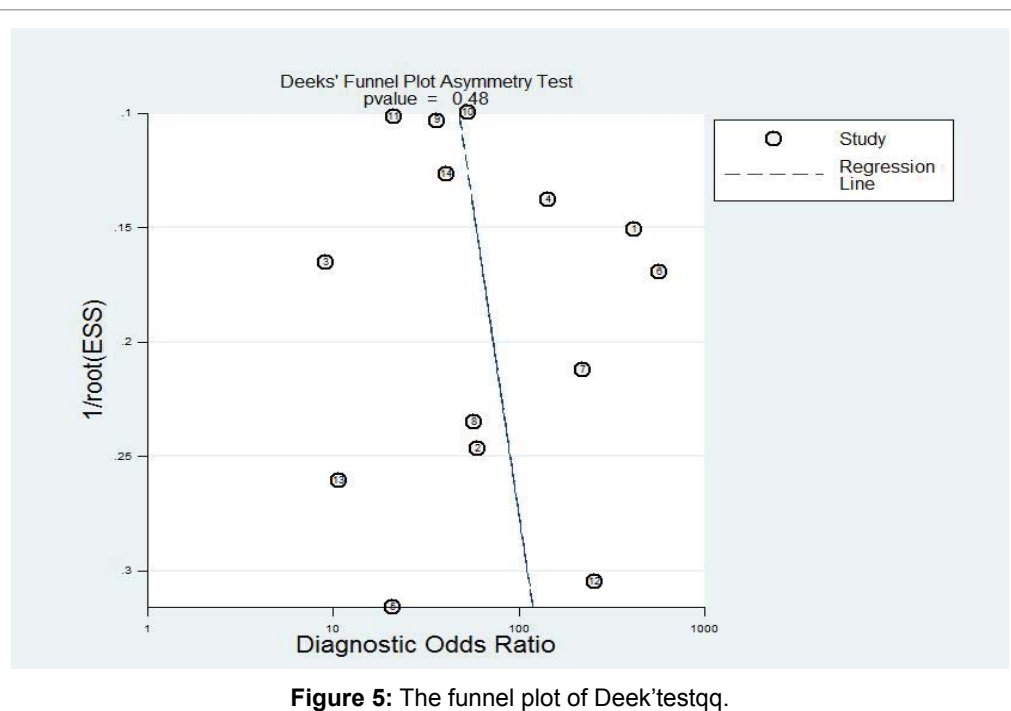

bias is another important factor in the reliability of meta analysis results. In this paper, Deek's funnel plot results indicated that there is no publication bias, which further indicates that ours results of this study are more reliable.

\section{Conclusion}

This meta-analysis suggested that $68 \mathrm{Ga}-\mathrm{PET}$ or PET/CT have higher sensitivity and specificity by detecting primary and metastatic lesions to diagnose neuroendocrine tumour.

\section{References}

1. Yao JC, Hassan M, Phan A, Dagohoy C, Leary C, et al. (2008) One hundred years after "carcinoid": Epidemiology of and prognostic factors for neuroendocrine tumors in 35,825 cases in the United States. Journal of Clinical Oncology Official Journal of the American Society of Clinical Oncology. 26: 3063-3072.

2. Li F (2017) 68Ga-radiopharmacueticals development: Advances and opportunities of nuclear medicine. Chin J Nucl Med Mol Imaging. 37: 129-131.
3. Whiting PF, Rutjes AW, Westwood ME, Mallett S, Deeks JJ, et al. (2011) QUADAS-2: A revised tool for the quality assessment of diagnostic accuracy studies. Ann Intern Med, 155: 529-536.

4. Zamora J, Abraira V, Muriel A, Khan K, Coomarasamy A (2006) Meta-DiSc: A software for meta-analysis of test accuracy data. BMC Med Res Methodol 6: 31

5. Gabriel M, Decristoforo C, Kendler D, Dobrozemsky G, Heute D et al. (2007) 68Ga-DOTA-Tyr3-octreotide PET in neuroendocrine tumors: Comparison with somatostatin receptor scintigraphy and CT. J Nucl Med : 48508-48518.

6. Naswa N, Sharma P, Kumar A, Hasan Nazar A, Kumar R, et al. (2011) Gallium68-DOTA-NOC PET/CT of patients with gastroenteropancreatic neuroendocrine tumors: A prospective single-center study. AJR Am J Roentgenol 197: 1221-1228.

7. Buchmann I, Henze M, Engelbrecht S, Eisenhut M, Runz A et al. (2007) Comparison of 68 Ga-DOTATOC PET and 111 In-DTPAOC (Octreoscan) SPECT in patients with neuroendocrine tumours. European Journal of Nuclear Medicine \& Molecular Imaging 34: 1617-1626.

8. Beiderwellen KJ, Poeppel TD, Hartung-knemeyer V, Buchbender C, Kueh $\mathrm{H}$ et al. (2013) Simultaneous 68Ga-DOTATOC PET/MRI in patients with gastroenteropancreatic neuroendocrine tumors: Initial results. Invest Radiol 48: 273-279. 
Citation: Liu X, Ran Q, Wei Z, Zhou S, Jiang T, et al. (2018) The Diagnostic Performance of 68Ga-PET or PET/CT in Neuroendocrine Tumour, A Meta Analysis. J Nucl Med Radiat Ther 9: 364. doi: 10.4172/2155-9619.1000364

9. Schraml C, Schwenzer NF, Sperling O, Aschoff P, Lichy MP et al. (2013) Staging of neuroendocrine tumours: Comparison of [68Ga] DOTATOC multiphase PET/ CT and whole-body MRI. Cancer Imaging13: 63-72.

10. Venkitaraman B, Karunanithi S, Kumar A, Khilnani GC, Kumar R (2014) Role of 68 Ga-DOTATOC PET/CT in initial evaluation of patients with suspected bronchopulmonary carcinoid. European Journal of Nuclear Medicine and Molecular Imaging 41: 856-864.

11. Srirajaskanthan R, Kayani I, Quigley AM, Soh J, Caplin ME, et al.(2010) The role of $68 \mathrm{Ga}$-DOTATATE PET in patients with neuroendocrine tumors and negative or equivocal findings on 111 In-DTPA-octreotide scintigraphy. Journal of Nuclear Medicine 51: 875-882.

12. Łapińska G, Bryszewska M, Fijołek-Warszewska A, Kozłowicz-Gudzińska I, Ochman $\mathrm{P}$, et al. (2011) The diagnostic role of 68Ga-DOTATATE PET/CT in the detection of neuroendocrine tumours. Nuclear Medicine Review Central and Eastern Europe 14: 16-20.

13. Naswa N, Sharma P, Gupta SK, Karunanithi S, Mohan Reddy R, et al. (2014) Dual tracer functional imaging of gastroenteropancreatic neuroendocrine tumors using 68Ga-DOTA-NOC PET-CT and 18F-FDG PET-CT: Competitive or complimentary?. Clin Nucl Med 39: 27-34

14. Haug AR, Cindea-Drimus R, Auernhammer CJ, Reincke M, Beuschlein F et al. (2014) Neuroendocrine tumor recurrence: diagnosis with 68Ga-DOTATATE PET/CT. Radiology 270: 517-525.
15. Versari A, Camellini L, Carlinfante G, Frasoldati A, Nicoli F et al. (2010) Ga68 DOTATOC PET, endoscopic ultrasonography, and multidetector CT in the diagnosis of duodenopancreatic neuroendocrine tumors. Clin Nucl Med 35: 321-328.

16. Ruf J, Schiefer J, Furth C, Kosiek O, Kropf S, et al. (2011) 68Ga-DOTATOC $\mathrm{PET} / \mathrm{CT}$ of neuroendocrine tumors: Spotlight on the CT phases of a triplephase protocol. J Nucl Med, 52: 697-704.

17. Mayerhoefer ME, Schuetz M, Magnaldi S, Weber M, Trattnig S, et al. (2011) Are contrast media required for (68)Ga-DOTATOC PET/CT in patients with neuroendocrine tumours of the abdomen. Eur Radiol 22: 938-946.

18. Haug AR, Cindea-Drimus R, Auernhammer CJ, Reincke M, Wangler B, et al. (2012) The role of $68 \mathrm{Ga}-\mathrm{DOTATATE}$ PET/CT in suspected neuroendocrine tumors. Journal of Nuclear Medicine Official Publication Society of Nuclear Medicine 53: 1686-1692.

19. John M, Meyerhof W, Richter D, Waser B, Schaer JC, et al. (1996) Positive somatostatin receptor scintigraphy correlates with the presence of somatostatin receptor subtype 2.Gut, 38: 33-39.

20. Graham MM, Gu X, Ginader T, Breheny P, Sunderland JJ, et al. (2017) 68GaDOTATOC Imaging of Neuroendocrine Tumors: A Systematic Review and Metaanalysis. J Nucl Med, 58: 1452-1458.

21. Treglia G, Castaldi P, Rindi G, Giordano A, Rufini V, et al. (2012) Diagnostic performance of Gallium-68 somatostatin receptor PET and PET/CT in patients with thoracic and gastroenteropancreatic neuroendocrine tumours: a metaanalysis. Endocrine 42: 80-87. 